\title{
Precise Positioning of Terfenol-D Actuator by Eliminating the Heat Generated by Coil
}

\author{
M. Ahanpanjeh ${ }^{1}$, M. Ghodsi ${ }^{2} \&$ Y. Hojjat ${ }^{1}$ \\ ${ }^{1}$ Department of mechanical engineering, Tarbiat Modares university, Tehran, Iran \\ ${ }^{2}$ Department of Mechanical and Industrial Engineering, Sultan Qaboos university, Muscat, Omn \\ Correspondence: M. Ahanpanjeh, Department of mechanical engineering, Tarbiat Modares university, Tehran, \\ Iran. E-mail: maryam.ahan@gmail.com
}

Received: July 7, 2016

doi:10.5539/mas.v10n9p232
Accepted: July 28, 2016

Online Published: August 26, 2016

\begin{abstract}
Large displacement, large generated stress and easy fabrication process lead to the large industrial use of the magnetostrictive materials. However, the thermal expansion restricts their application in precise positioning, which is about $12 \mathrm{ppm} /{ }^{\circ} \mathrm{C}$ and too much to be neglected. In this research, the cooling system was exploited in order to eliminate the joule heat generated by coils under the direct current and solve inaccuracy problem. The investigation consists of two parts; first was design and fabricating the actuator of Terfenol-D with length of $30 \mathrm{~mm}$ which offers $10 \mu \mathrm{m}$ displacement under the DC current of $1 \mathrm{~A}$, and second was the cooling system consisting of the thermoelectric cooler, heat sink and control circuit to inhibit the Terfenol-D temperature to be increased. Without the cooling system, as the time was going on and the Terfenol-D was warming up, after about two hours the displacement reached to $68 \mu \mathrm{m}$ which deteriorated the accuracy of positioning significantly. By applying the cooling system, the displacement was maintained in $10 \mu \mathrm{m}$ with the tolerance of $\pm 1 \mu \mathrm{m}$, which realized the precise positioning by the Terfenol-D actuator.
\end{abstract}

Keywords: Terfenol-D actuator, precise positioning, thermoelectric cooler, temperature control

\section{Introduction}

Recently, needs of precise positioning in developing the systems acting at the micro- or nano- scale, have been increased in various industrial fields such as ultra-precision machine tools, micro motors, micro pumps, robotics, biomedical engineering, etc. In order to generate precise displacements piezoelectric actuators are widely used due to the high resolution and high bandwidth that they can offer. On the other hand, they have some restrictions which make magnetostrictive materials be more efficient. GMM has the output power several times and the displacement several ten times more than PZT. In general, GMMs have some special characteristics in comparison with PZTs which make them efficient functional material in actuators and sensors. These properties are as following: 1) large displacement, 2) large generated stress, 3) high Curie temperature, 4) simple fabrication process, 5) remote actuation (noncontact driving) (Ho-Mun, 2004; Andani et al., 2014; Hadi et al., 2014; Moghaddam et al., 2014; Hayato \& Hiroshi, 2013).

For achieving the precise positioning, the different losses have to be taken into the account in designing the actuator. These losses are hysteresis losses, eddy current losses in active material and resistive losses in drive coil. Hysteresis and eddy current losses happen when the driving current is AC, however, the latter happens in both AC and DC currents. The heat generated by drive coil, known as Joule heat, is computed (Hyun-Mo, Yeon-Ho \& Song, 2013)

$$
P_{w}=\frac{1}{2 \sigma_{e}} \int J_{s}^{2} d v
$$

Where $P_{w}$ is the power loss in the coil winding [W], $\sigma_{e}$ is the electric conductivity $[\mathrm{S} / \mathrm{m}], J_{s}$ is the current density $[\mathrm{A} / \mathrm{m} 2], d v$ is the incremental volume element $\left[\mathrm{m}^{3}\right]$. Since the precise positioning in actuator is objective, the direct current is applied, thereby the only loss affecting the accuracy of positioning is Joule heat. 
By increasing the temperature of the driving coil, Terfenol-D expands and regarding to the expansion coefficient which is $12 \mathrm{ppm} /{ }^{\circ} \mathrm{C}$ (Grunwald, 2008) for Terfenol-D, the accuracy of actuator significantly demolishes. Eda et al. (Eda, Sahashi, Kobayashi \& Ikawa, 1992) applied a water cooling device in GMM actuator for an ultraprecision machine to solve the joule heat problem. The cooling water device was made of copper tube of diameter $2 \mathrm{~mm}$ and incorporated to isolate the heat between the driving coil and magnetostrictive rod. Kellog (Kellogg, 2000), in order to eliminate the joule heat, designed and fabricated water-cool transducer such that Terfenol-D rod was located in the center and immediately surrounding the rod was a drive coil, and the cooling tubes were encased exterior to the drive coil. At the same time, he also designed air-cooled transducer so that Terfenol-D rod resided at the center and immediately surrounding the rod an air gap was located which let cooling air-flow be passing through. These models had some disadvantages, such as the big size of transducer, decreasing the cooling capacity of fluid over time, pumping fluid, etc. In this paper, thermoelectric cooler was applied for overcoming these problems and eliminating the heat generated by coil. TEC has no moving part making it vibration- and noise- free element. Cooling is achieved by passing the current through the thermoelectric materials, and the magnitude of the current specifies the rate of cooling (Annapragada, Kolodner, Marc \& Suresh, 2012; Esfahani et al., 2016; Moghaddam et al., 2016; Raad, Moghaddam \& Elahinia, 2016 ), which leads to precise temperature control.

\section{Design, Analysis and Manufacturing}

\subsection{Design and Analysis of the Actuator}

Figure 1 shows the cooling layout of the actuator schematically. The cold side of TEC is attached to the actuator and the hot side is connected to the heat sink and fan. In that case, the heat generated by the coils is absorbed by the TEC and released to the ambient through the heat sink and fan.

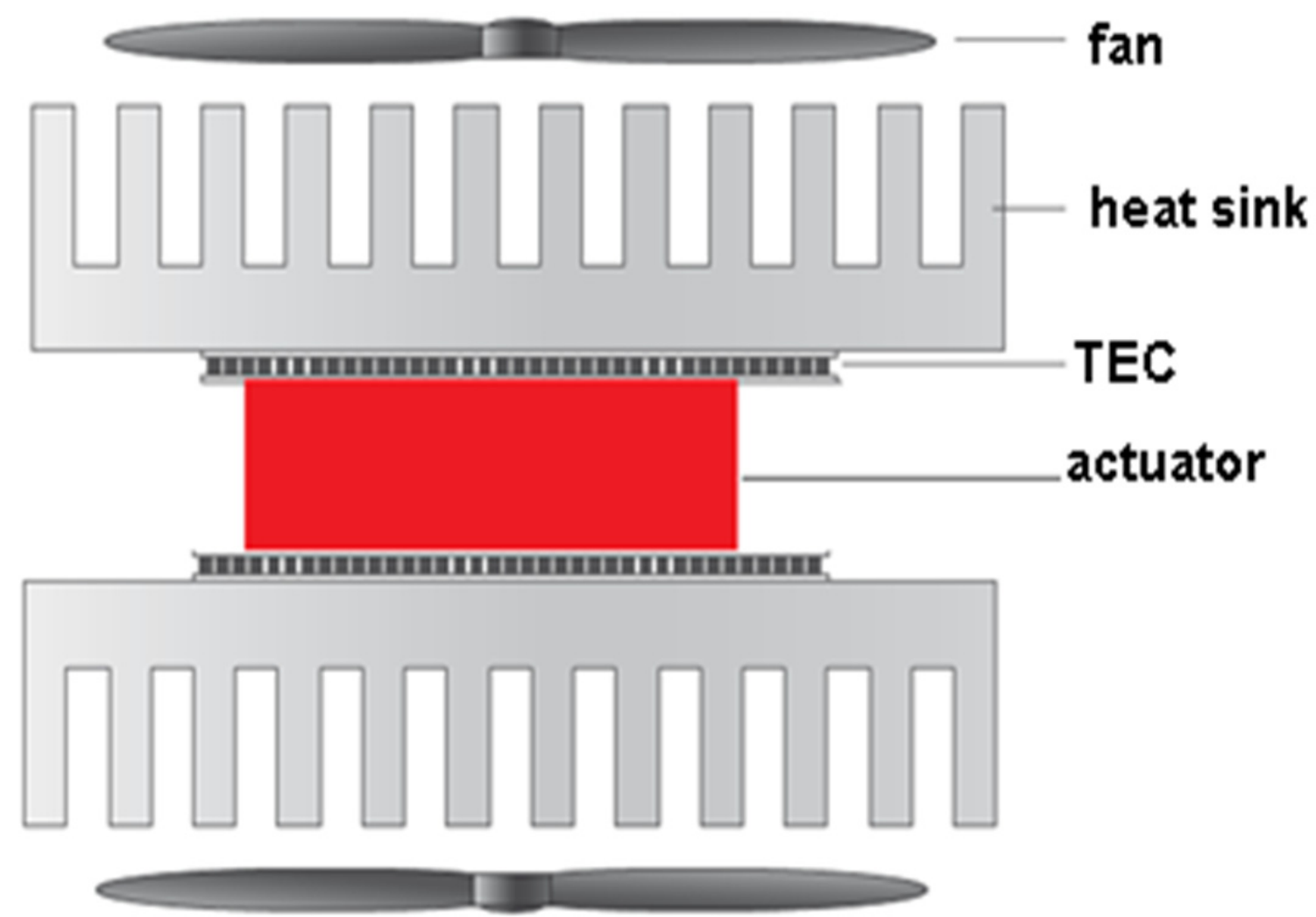

Figure 1. Schematic cooling layout of the actuator

The magnetostrictive actuator has been designed based on known dimensions of Terfenol-D with the rectangular cross- section (each side $2 \mathrm{~mm}$ and length $30 \mathrm{~mm}$ ) and simulated in ANSYS FEA. The task of optimization of the design was achieved with respect to various aspects like magnetic, thermal and structural simultaneously. For an 
improved accuracy, the indirect method was applied for coupling magnetic, thermal and structural analysis so that the result of magnetic analysis became loads for the thermal analysis and the thermal result became loads for the structural analysis. The structure of magnetostrictive actuator in simulations is shown in fig. 2. In this structure, Terfenol-D was implanted at the center and surrounded by the permanent magnets which provide a magnetic bias field and help to achieve desirable functionality and linear response, and the driving coil was located surrounding the magnets and excited by the DC current. TMC (Grunwald, 2014) (Terfenol-D- magnetcoil) layout was chosen for a couple of reasons. Firstly, in that case, there is a maximum distance between Terfenol-D and coil. Also, coil is in the adjacent of the thermoelectric cooler which leads to the efficient cooling.

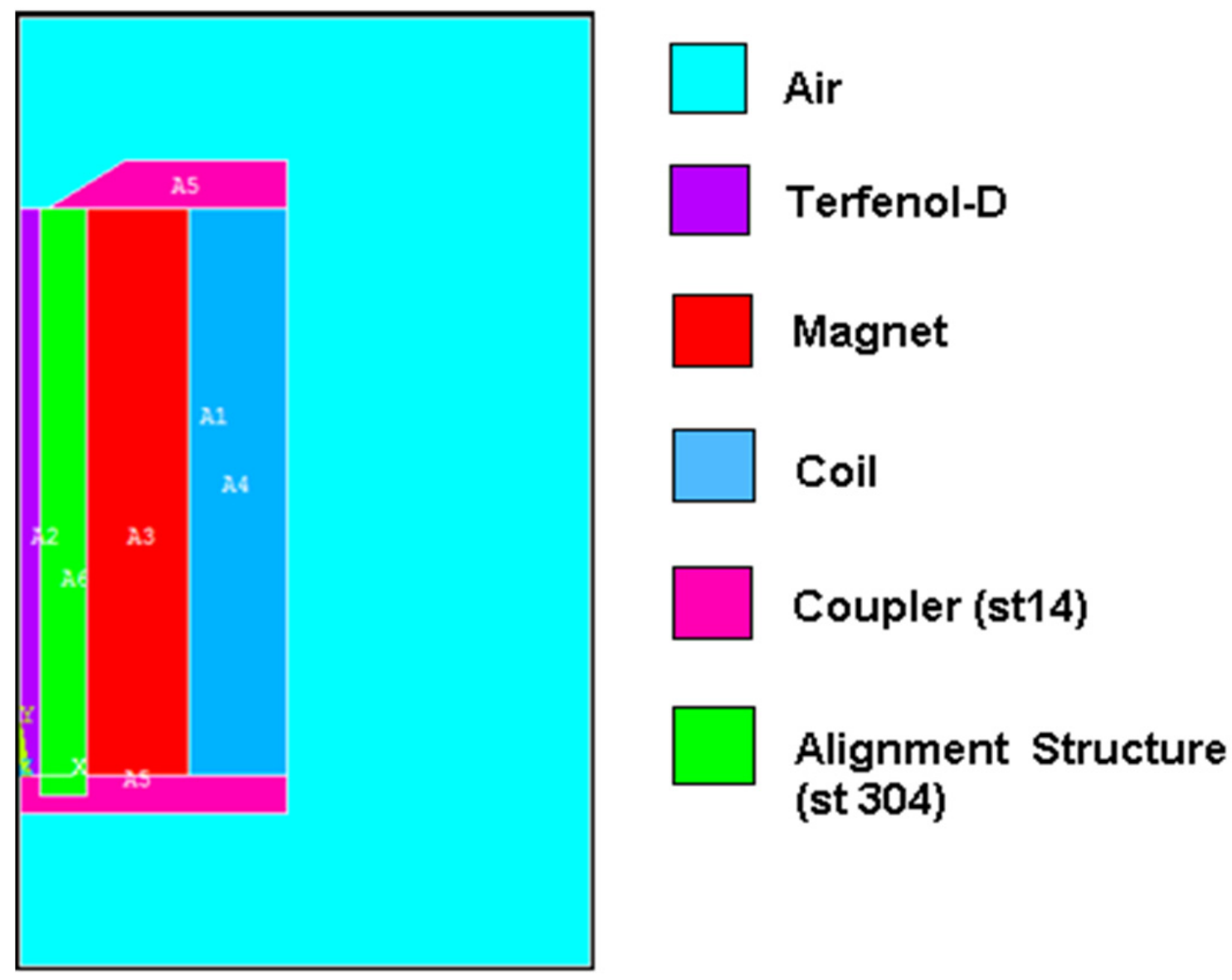

Figure 2. Terfenol-D actuator model in Ansys

The distribution of magnetic flux in the magnetostrictive rod is an important factor has to be considered in the design of the actuator. The uniformity and linearity of magnetic intensity determines the sensitivity and linearity of the output motion (Wang, Liu \& Yao, 2006). In the simulations, the number of coils is 600 and the applied current density is $j=4 \times 10^{6} \mathrm{~A} / \mathrm{m}^{2}$. Figure 3 shows the total magnetic flux density caused by permanent magnets and coil. The distribution of the magnetic flux is uniform which means that the displacement coefficient is almost constant in every points of the magnetostrictive rod. In the present design, Terfenol-D reaches to saturation point. Fig. 4 shows the Joule heat generated by the coil under the direct current and is described as below. Where $I$ is the electrical current $[\mathrm{A}]$ and $\rho$ is the electrical resistivity $[\Omega]$.

$$
Q_{\text {joule }}=I^{2} \rho
$$




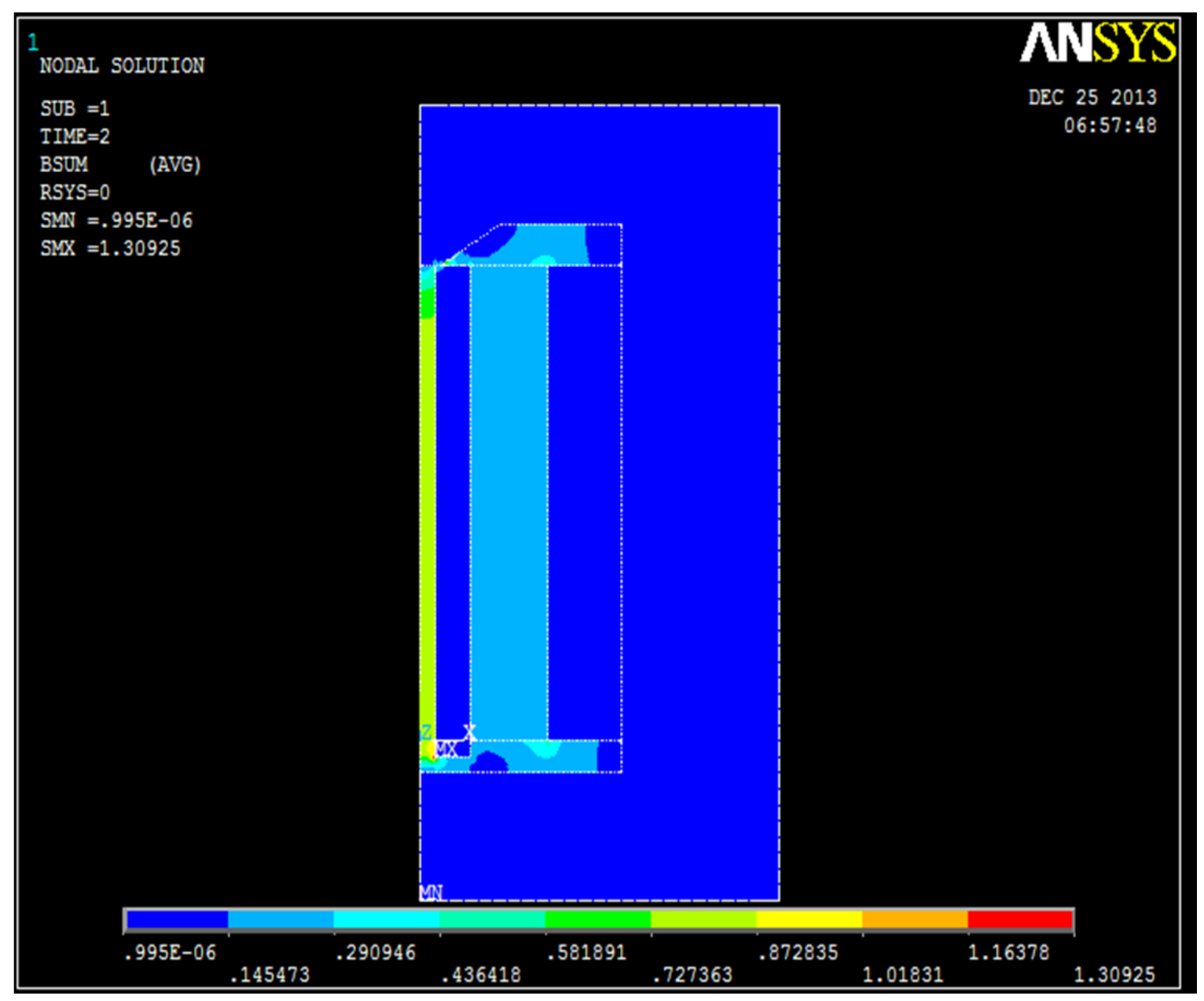

Figure 3. Total flux density Distribution 


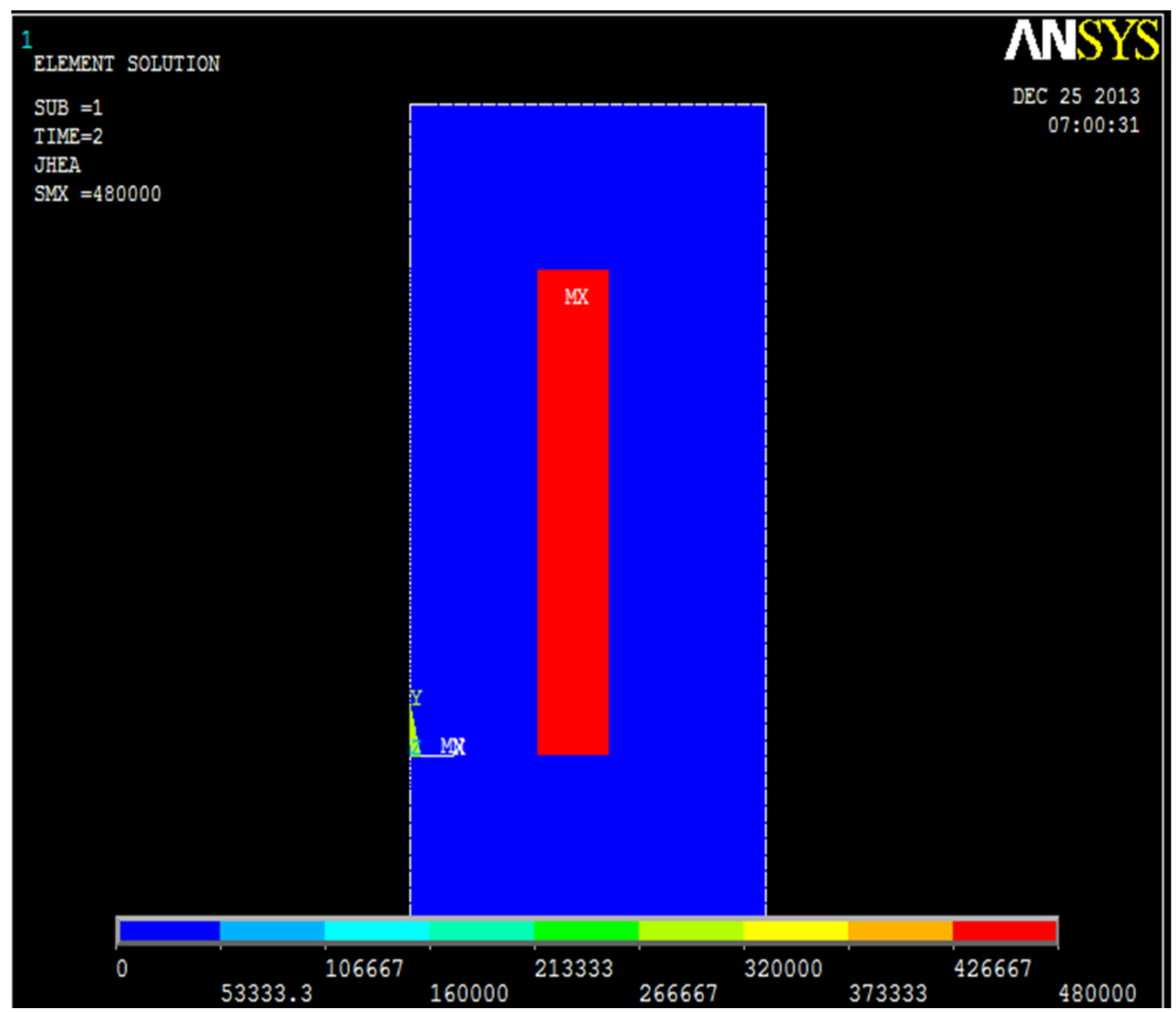

Figure 4. Joule heat generated by the coil

For the better and precise result, 3D analysis is done in thermal and structural environments. By loading 480000 $w / m^{3}$ Joule heat and considering the convection coefficient $h_{w}=9.17 \mathrm{w} / \mathrm{m}^{2} \mathrm{~K}$, temperature distribution in the actuator is obtained as a Figure 5 . 


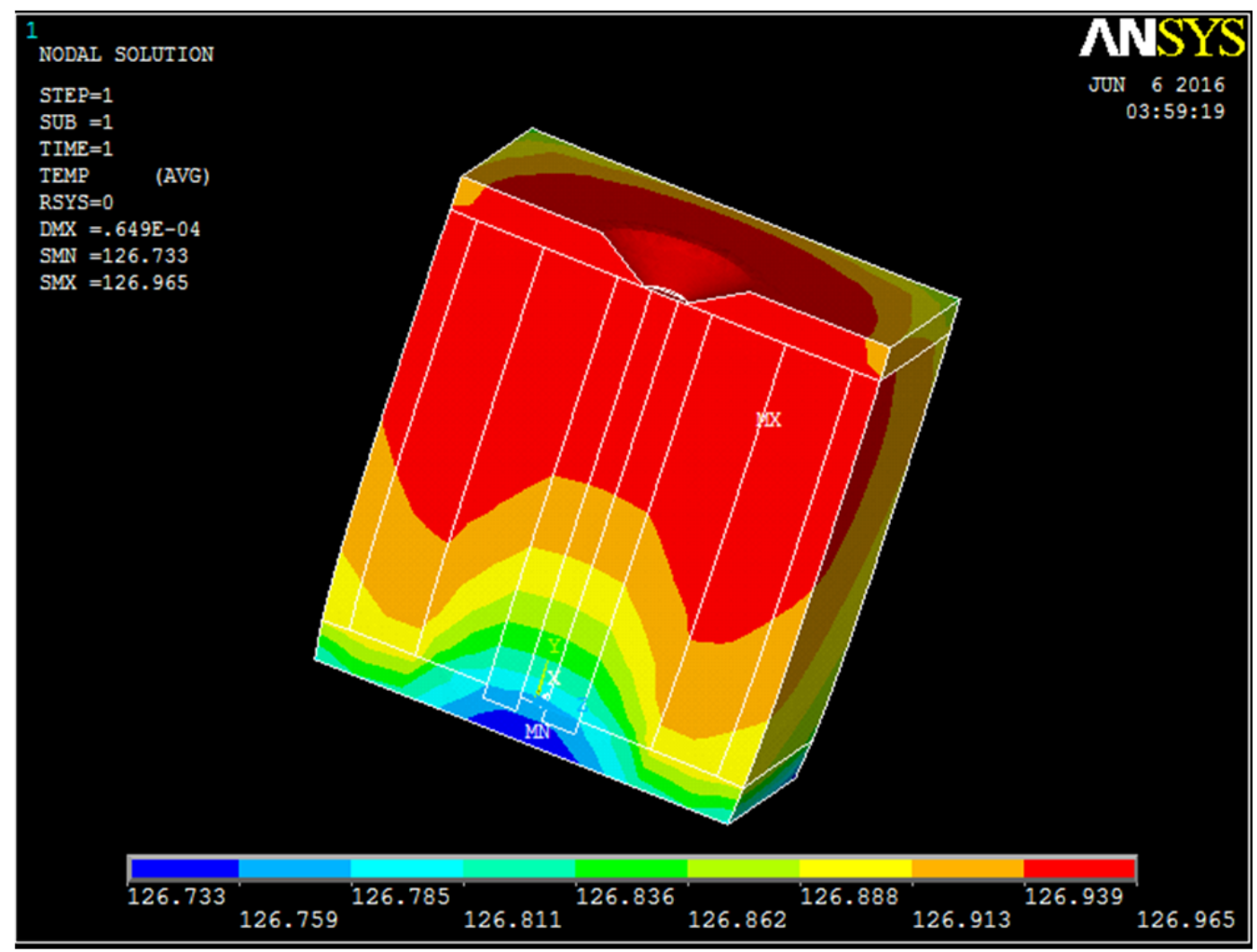

Figure 5. Temperature distribution in the actuator

As Figure 5 shows, the temperature rises from 20 up to 126.965 in steady state analysis. The results received from thermal analysis are loaded into the structural analysis and the heat expansion of Terfenol-D is concluded as Figure 6.

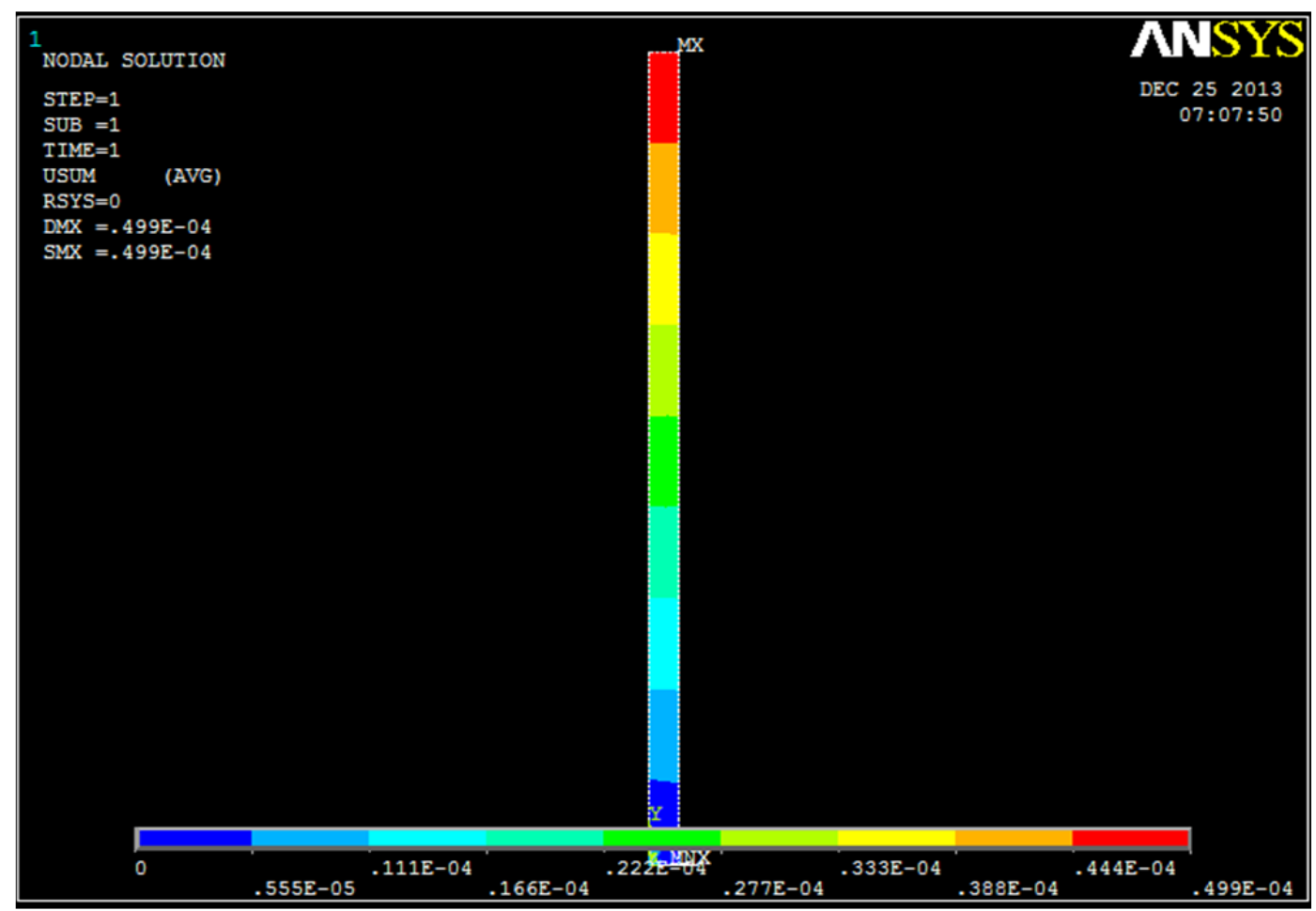

Figure 6. Displacement of the Terfenol-D caused by the joule heat 
For understanding the rate of heat expansion, transient analysis was also done. Fig. 7 depicts the rate of increasing the temperature in Terfenol-D. According to the analysis, the system needs almost two hours to reach the steady state position.

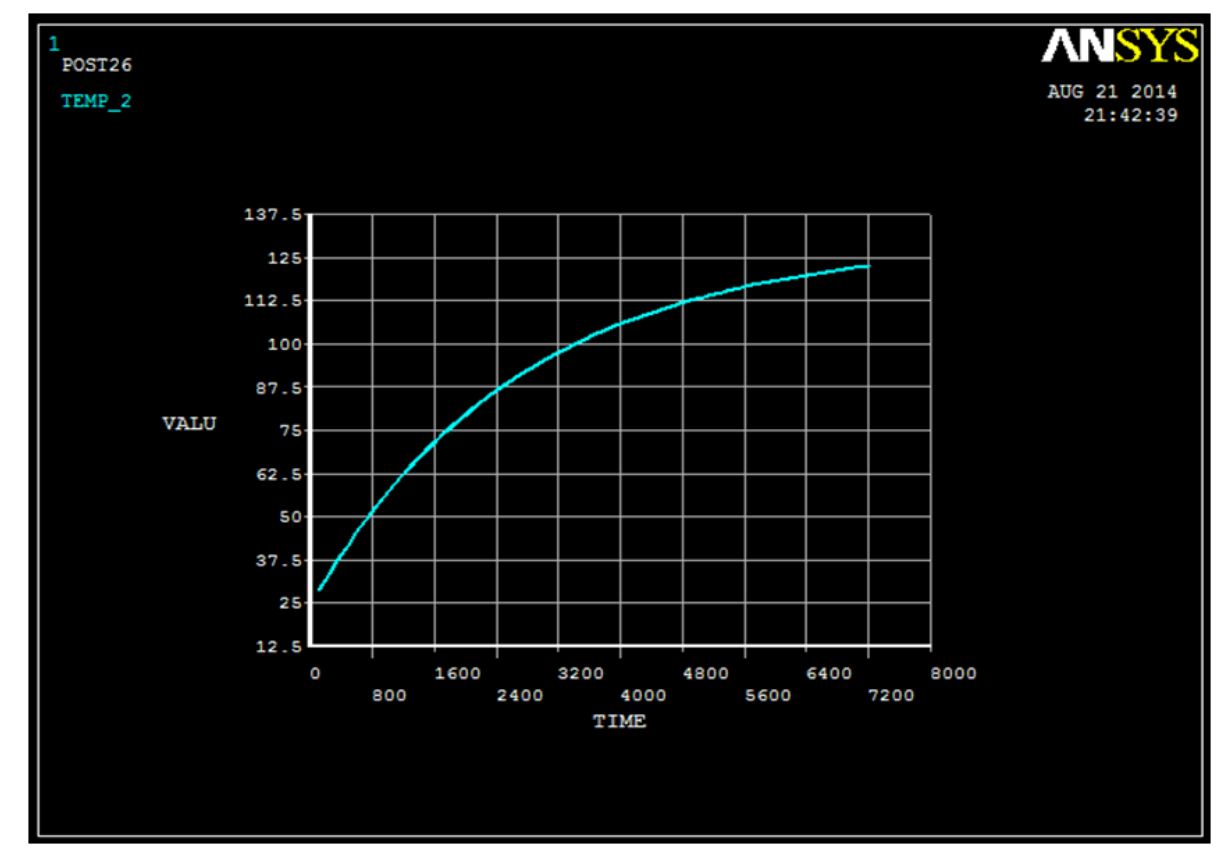

Figure 7. The rate of increasing the temperature in Terfenol-D

\subsection{Manufacturing of the Actuator}

According to the Ansys analysis, the actuator was designed and manufactured. The couplers were machined and the groove was generated by Spark, so the Terfenol-D can be located on the bulge. The alignment component was machined, and the rectangular hole was generated by wire cut. The copper case was manufactured by the lathe. Fig. 8 shows a) actuator's components, b) the actuator.

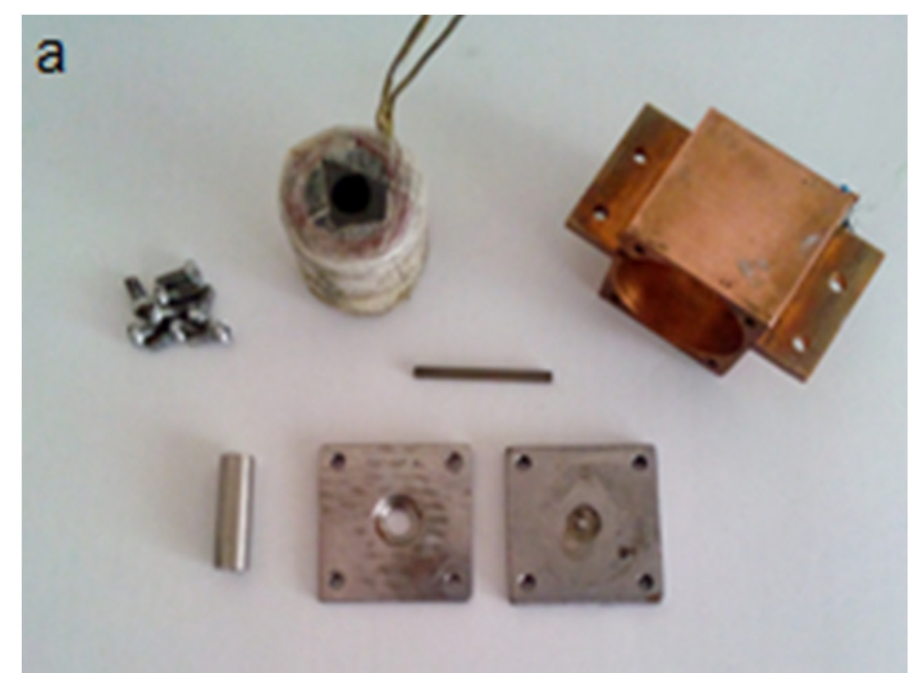




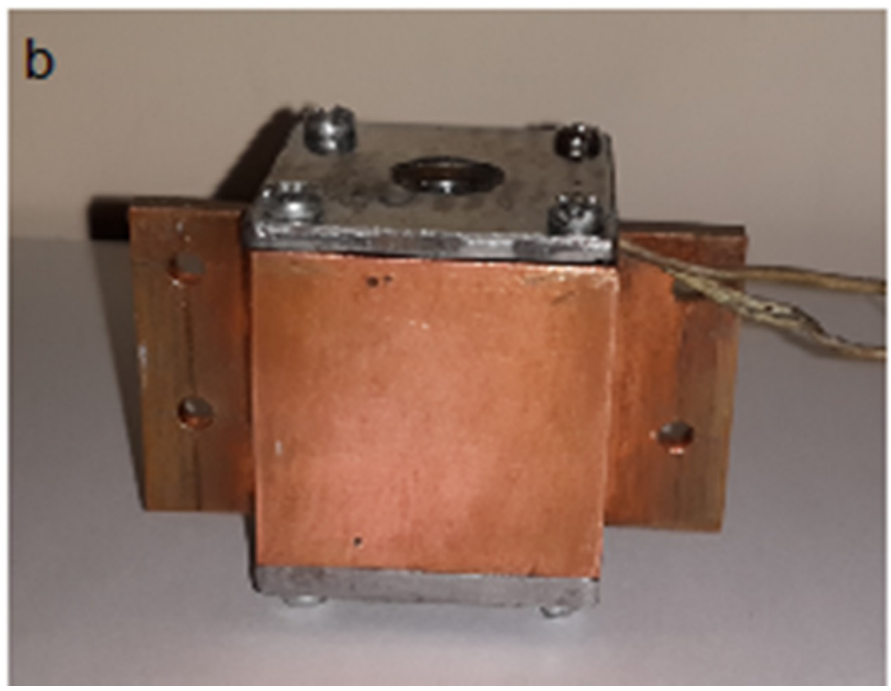

Figure 8. a) The components and b) the Terfenol-D actuator

\subsection{Design and Analysis of TEC}

As the thermal performance of thermoelectric cooler depends on the sufficient dissipation of the heat from the hide side, the design of heat sink should go under consideration. The heat released from the actuator is absorbed by the cold side of a thermoelectric cooler, and pumped to the hot side. The pumped heat with the input power to the cooler is then extricated to the ambient through a heat sink (Huang \& Duang, 2000). $Q_{L}$, cooling load, which is conducted from the cold side to the hot side, should be greater than the heat generated by the coil for the proper cooling. The further simulations are required in order to selecting the sufficient thermoelectric cooler and heat sink. For the simulations, the temperature of the hot and the cold sides should be determined first as the boundary conditions. A simple experiment was done to get these temperatures by selecting TEC 12704, with 127 pair of semiconductor elements, the Aluminum heat sink $(77 \mathrm{~mm} \times 67 \mathrm{~mm}$, fin thickness $=0.6$, fin length $=30 \mathrm{~mm}$ and fin number=25) and the fan with 4.8 watt power. By applying the current of $3.3 \mathrm{~A}$ (determined in datasheet as a maximum value) to TEC the temperatures of the hot and the cold side of the TEC were extracted $62^{\circ} \mathrm{C}$ and $4^{\circ} \mathrm{C}$ respectively. Ansys analysis was run according to the experimental data. The temperature distribution and the value of the cooling load were shown as a fig. 9. $Q_{L}$ was obtained $-13.802 \mathrm{~W}$, minus sign indicates that the heat released from the hot side. According to the previous analysis, the joule heat was obtained $480000 \mathrm{w} / \mathrm{m}^{3}$ which in the selected coil leads to:

$$
480000 \times \pi\left(14^{2}-9^{2}\right) \times 30 \times 10^{-9}=5.2 \mathrm{~W}
$$

Since cooling load of the thermoelectric cooler is bigger than the amount of joule heat generated by the coil, the selected TEC, fan and heat sink is satisfying. 


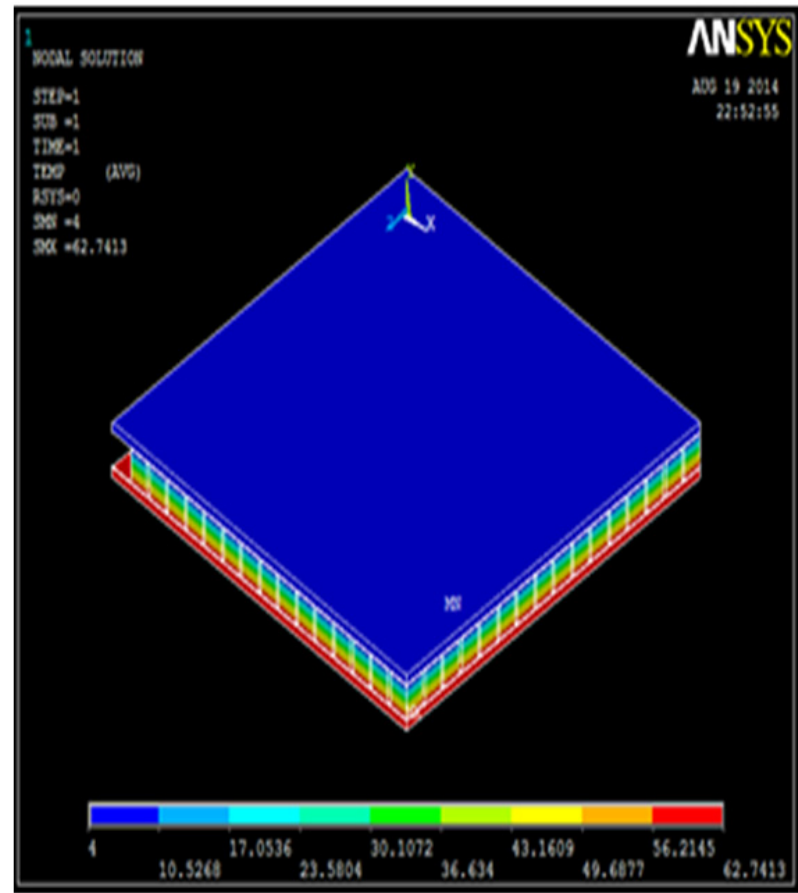

b

Figure 9. a) The temperature distribution in TEC, b) value of the cooling load $\left(Q_{L}\right)$

\section{Temperature Control of the Actuator}

Since the purpose of this paper is the precise positioning of actuator in micro scale, the efficient feedback controller should be exploited. The temperature of the Terfenol-D needs to be maintained at a constant and stable value near to the room temperature under variable currents applied to the driving coils. Two TEC with two heat sink and two fans are located at the two sides of the copper case and a thermally-conductive silicon paste are applied on the heat and cold sides of the TEC to minimize contact resistances. Since the driving coils are surrounded by the copper case, the generated heat will be absorbed and released through the TEC to the ambient before affecting the temperature of the Terfenol-D. By maintaining the temperature of the copper case at the constant value, the Terfenol-D temperature is almost controlled. The temperature sensor SMT 160 is mounted to the copper case. SMT160 is a three- terminal integrated temperature sensor with the output signal in pulse width form. Due to the nonlinearities of the system (consisting of TEC, heat sink and fan, which are connected to the hot side, and the actuator connected to the cold side), deriving the mathematical model is not that simple. For a better controller design, the dynamic model of the system should be known first, therefore a computational intelligent system identification techniques in MATLAB has been adopted to understand the dynamic behavior of the system. Parametric system identification is a method of control and prediction of a real physical system, which is defined by the model structure and parameters from input and output data of the system to describe the real physical system (Nurul, Nurul \& Toha, 2013) (Huang, 2000). The input data of the system was variable values of voltage and the output data was corresponding temperature of actuator taken with the SMT160. For having the measurements of the input and the output, PIC18F452 was exploited as a microcontroller and the written program was compiled in MicroC software. It is so important to make the collected data almost continuous in an appropriate sampling time to represent the actual system. The input voltage varying from zero up to $11.2 \mathrm{v}$ with the small increment of $0.05 \mathrm{v}$ and the sampling time of 3 seconds, was applied to the system by PWM of the microcontroller and the corresponding temperatures were taken by the temperature sensor, and both recorded at the computer. In order to filter the noises, a filter with the cutoff frequency of $72.34 \mathrm{HZ}$ was used before recording the data. Figure 10 shows the voltage and corresponding temperature data of the system. 


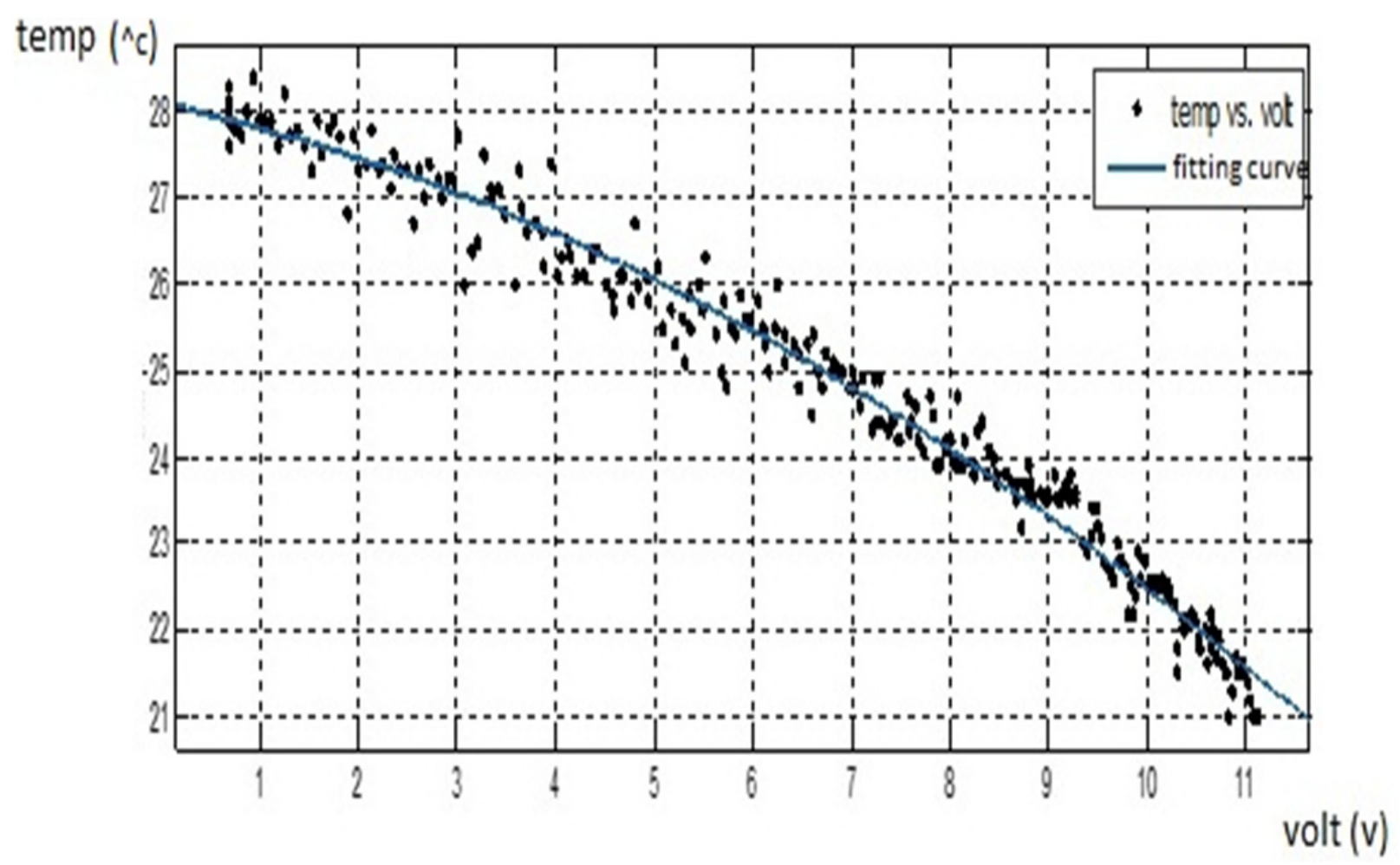

Figure 10. System's temperature behavior via voltage inputs

These particular input voltages and output temperatures were then loaded to the system identification system toolbox in MATLAB in order to define the transient behavior of the system. It is crucial to derive the mathematical model of the system with the proper order because improper lower order model may bode that the mathematical model is inadequate, while improper higher order model may augments the uncertainties. Due to the sampled experimental data, the discrete time domain was exploited and the transfer function of the system is achieved in z-plane as:

$$
G(z)=\frac{-10.28 \times 10^{-5} z^{-1}}{1-1.051 \times z^{-1}-0.7246 z^{-2}+0.7759 z^{-3}}
$$

According to the slow dynamic of the system, TEC demands a low bandwidth controller. Simple ProportionalDerivative- Integral (PID) control almost suffices to gain precise closed-loop performance. The proportional term provides rapid closed-loop transient response to step changes in the temperature set point and fast rejection of thermal disturbances. The integral term provides zero steady- state error with a constant temperature set point and constant disturbances. The derivative term reduces transient overshoot and oscillation in the temperature when set point is changed from one value to another (Alter, 2003; Rahmanian et al., 2014; Shayesteh, 2015).

Based on the achieved transfer function of the system, PID terms were derived in Matlab PID tuner. The value of $\mathrm{P}, \mathrm{I}$ and $\mathrm{D}$ terms are $-1234,-0.8$ and zero respectively. As the set point is constant, PI controller engenders precise control. In this work, the control signal takes the form of pwm outputs from the PIC microcontroller and the set point is fixed at the $22^{\circ} \mathrm{C}$. Therefore the temperature of the actuator is maintained at this value with the tolerance of $\pm 0.5^{\circ} \mathrm{C}$.

\section{Results and Discussion}

In this experiment, the purpose was determination of the displacement of the Terfenol-D under the current of $1 \mathrm{~A}$ (the maximum value can be applied to the driving coils). For measuring the displacement of the actuator, laser gap sensor with the accuracy of $1 \mu \mathrm{m}$ was exploited. At the beginning, Terfenol-D actuator generates $10 \mu \mathrm{m}$ displacement to the response to the current of $1 \mathrm{~A}$, by continuing the given current, coils start to heat and following the rises in the temperature, the thermal expansion takes place, which deteriorates the accuracy of the actuator. The displacement of the actuator was taken every 30 seconds for two hours. Figure 11 shows the displacement of the actuator during this 2 hours. 


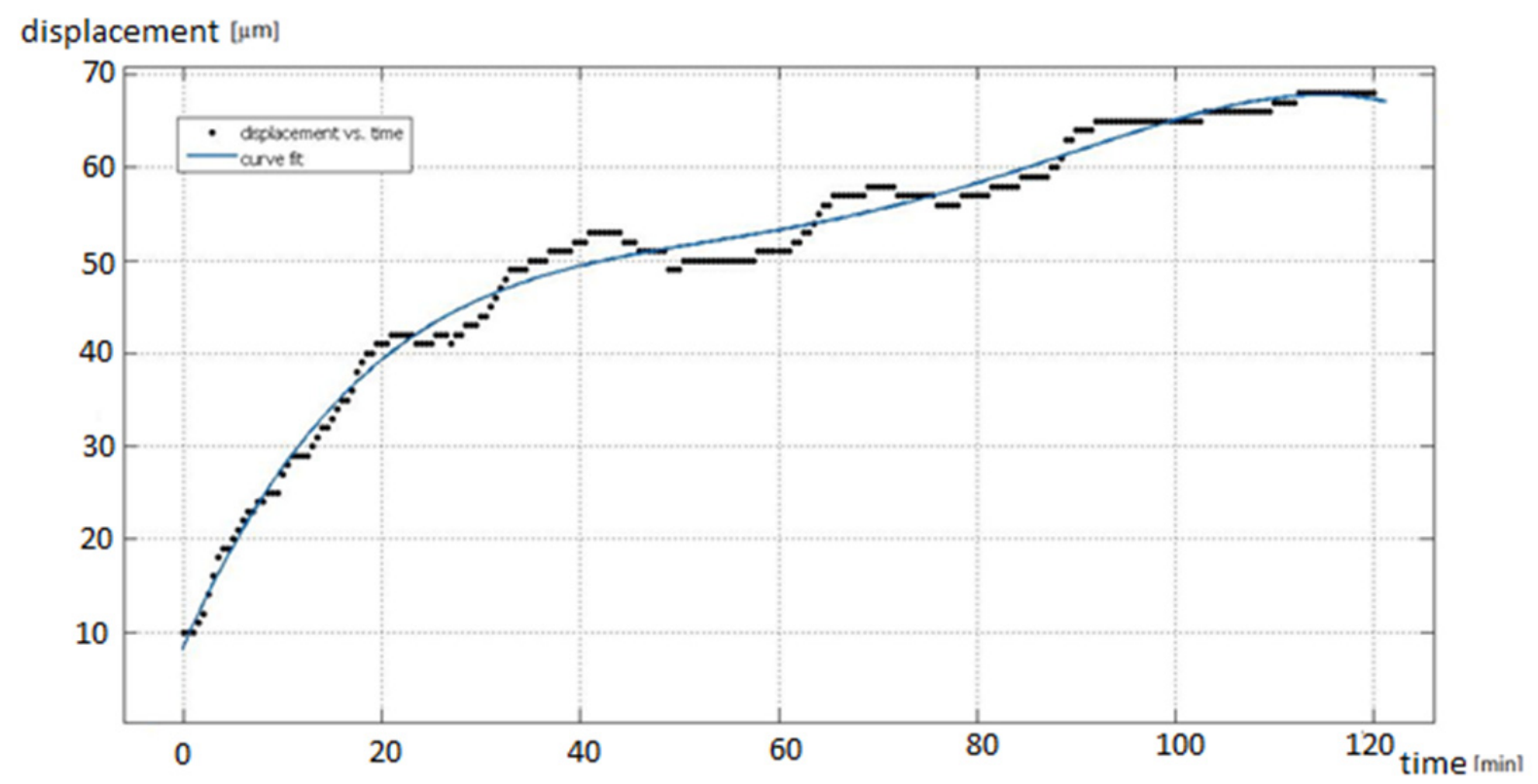

Figure 11. Thermal expansion of Terfenol-D in 2 hours

As the figure 11 shows, the actuator reaches to its steady states after about 2 hours, which is in accordance with Ansys analysis as shown in figure 7. The displacement of the actuator was 68 micrometer during 2 hours, that first 10 micrometer was because of the magnetostriction and 58 micrometer was the result of the heat expansion. The next experiment was done with the cooling system to reduce the heat expansion and improve the accuracy of the actuator. The measurements are done every 30 seconds, same as the previous measurements, for 1 hour, since the maximum temperature rise happens in the first minutes. Figure 12 shows the process of measurements along with the cooling system. Figure 13 shows the displacement of the actuator with and without the cooling system. By eliminating the heat expansion, the displacement of the actuator was remained in 10 micrometers with the tolerance of $\pm 1 \mu \mathrm{m}$ which was just because of the magnetostriction of Terfenol-D under the applied current of 1A. Thus the intention of precise positioning was realized.

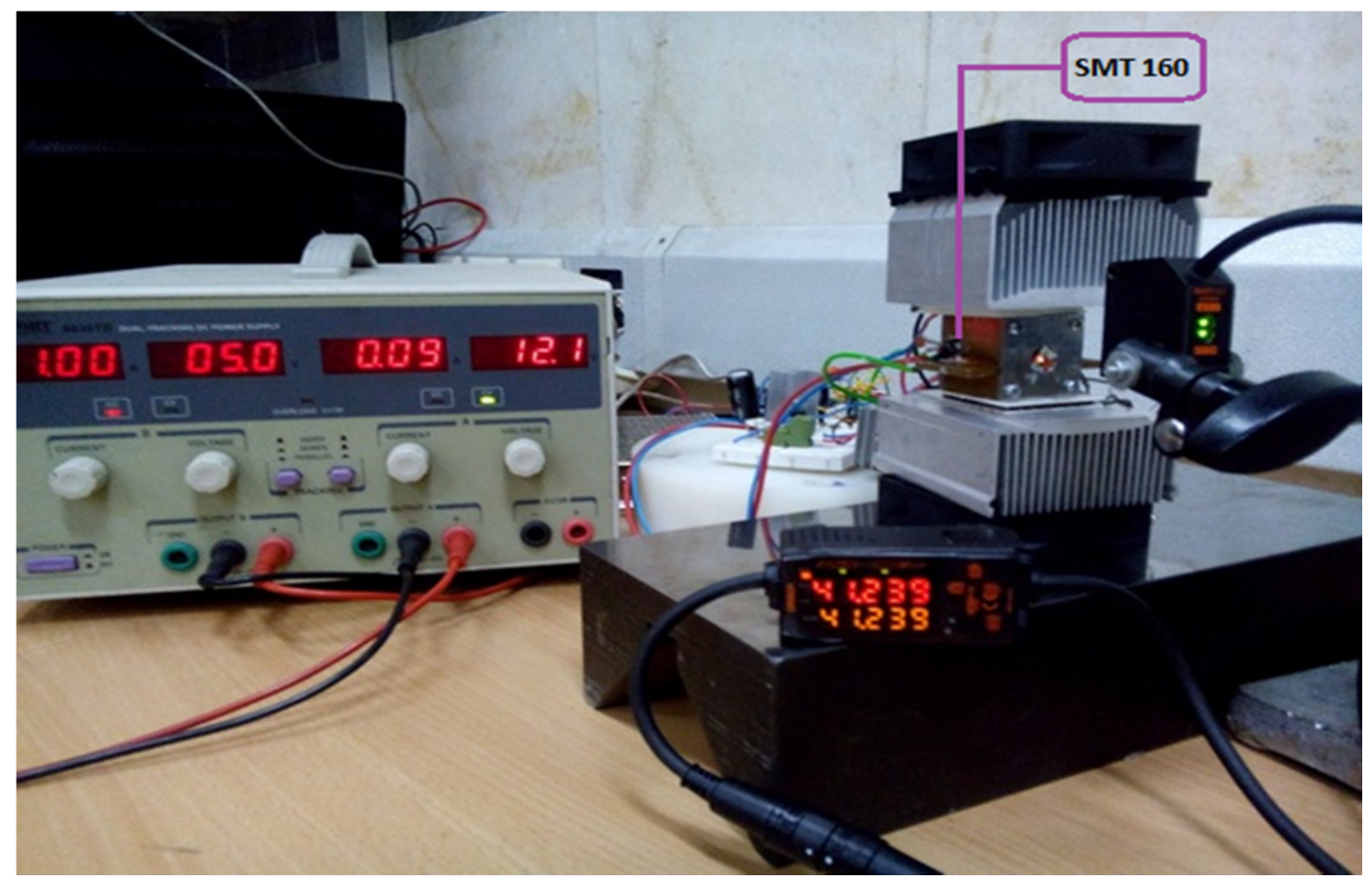

Figure 12. Process of measuring the displacement of the actuator connected to temperature control circuit 


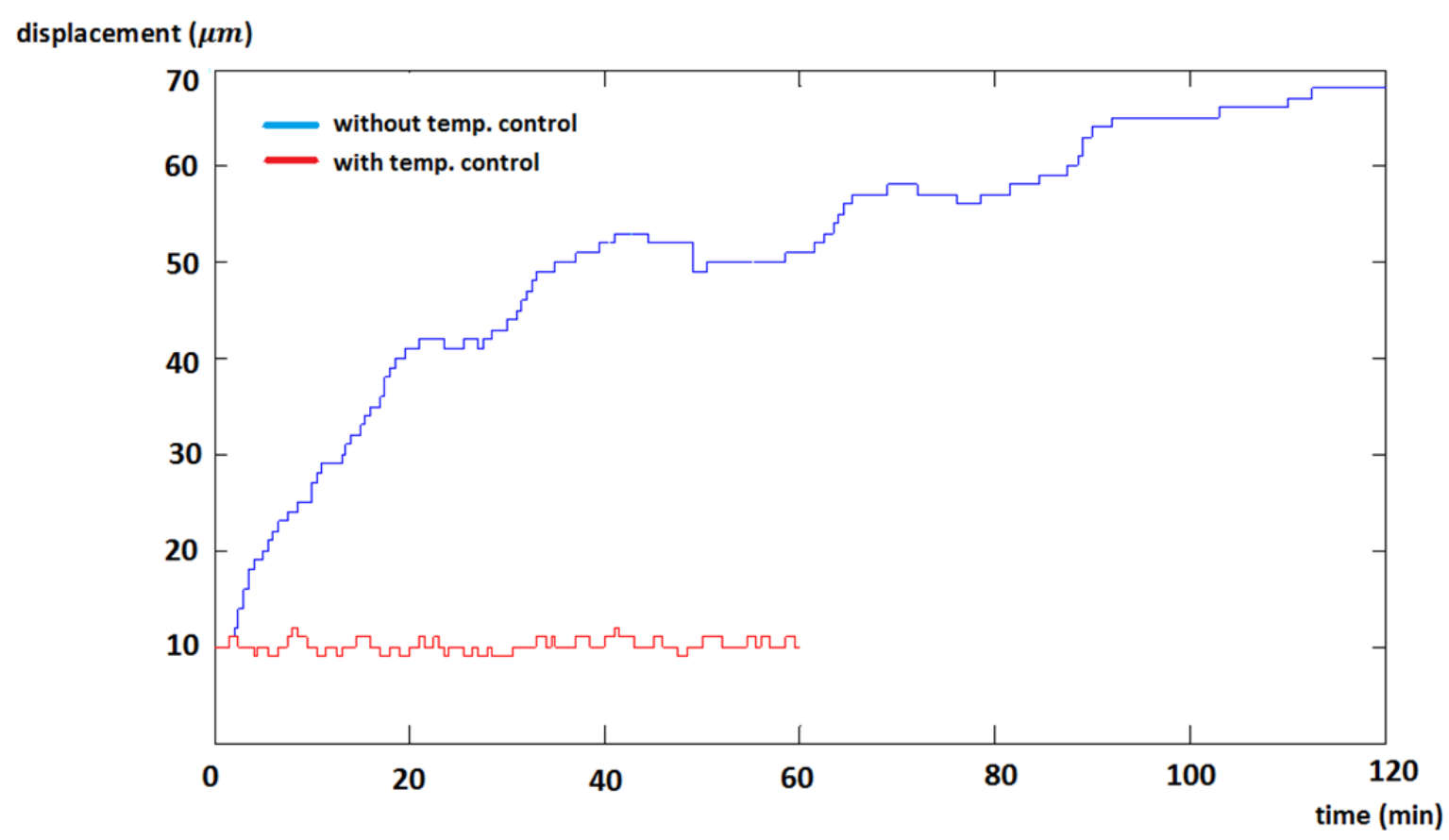

Figure 13. Comparison of the displacement of the actuator with and without the cooling system

\section{Conclusions}

This research consists of two main parts; Terfenol-D actuator and cooling system. The process of design and fabricating the actuator was so that the precise positioning could be realized. TMC layout was chosen for the actuator to provide the maximum distance between Terfenol-D and coil. In order to decrease the required current and consequently the joule heat for achieving the specified displacement, the permanent magnet was exploited. At the cooling system, the performance of TEC depends to the sufficient releasing of the heat from the hot side, so that the selection of the heat sink and fan was done carefully. In Ansys analysis the displacement caused by heat expansion was extracted $45 \mu \mathrm{m}$, however, in the measurements the displacement reached to $58 \mu \mathrm{m}$, which was enormously big Compared with magnetostriction, $10 \mu \mathrm{m}$. By applying the heat control system, the displacement of the actuator was dependent only to the magnetostriction and kept $10 \mu \mathrm{m}$ with the tolerance of $\pm 1 \mu \mathrm{m}$.

\section{References}

Alter, D. M. (2003). Thermoelectric Cooler Control Using a TMS320F2812 DSP and a DRV592 Power Amplifier. Texas Instruments: Dallas, Texas.

Andani, M. T. et al. (2014). Metals for bone implants. Part 1. Powder metallurgy and implant rendering. Acta biomaterialia, 10(10), 4058-4070.

Eda, H. E. O., Sahashi, M., Kobayashi, T., \& Ikawa, N. (1992). Ultraprecise machine tool equipped with a giant magnetostriction actuator development of new materials, Tb, Dy, -x (Fe,Mn, --y)n, and their application. CIRP Annals- Manufacturing Technology, 41, 421-424.

Esfahani, S. N. et al. (2016). Independent tuning of stiffness and toughness of additively manufactured titanium-polymer composites: Simulation, fabrication, and experimental studies. Journal of Materials Processing Technology, 238, 22-29.

Grunwald, A. A. G. O. (2008). Design of a magnetostrictive (MS) actuator. Sensors and Actuators A, 144, 161-175.

Grunwald, A. G. O. A. A. (2014). Design and Application of Magnetostrictive "MS" Materials. Dublin City University, School of Mechanical and Manufacturing Engineering: Dublin, Ireland.

Hadi, A. et al. (2014). Modeling and Experiment of a Flexible Module Actuated by Shape Memory Alloy Wire. in ASME 2014 Conference on Smart Materials, Adaptive Structures and Intelligent Systems. American Society of Mechanical Engineers. 
Hayato, Y. H. S., \& Hiroshi, S. (2013). A newly developed rotary-linear motion platform with a giant magnetostrictive actuator. CIRP Annals-Manufacturing Technology, 62, 371-374.

Ho-Mun, S. C. C. (2004). Finite element modeling of magnetostriction for multilayered MEMS device. Journal of Magnetism and Magnetic Materials, 270, 167-173.

Huang, B. J. C. J. C., \& Duang, C. L. (2000). A design method of thermoelectric cooler. International Journal of Refrigeration, 23, 208-218.

Huang, B. J. C. L. D. (2000). System dynamic model and temperature control of a thermoelectric cooler. International Journal of Refrigeration, 23, 197-207.

Hyun-Mo, A., Joe. K. K., Yeon-Ho, O., \& Song, K. D. (2013). Multi-physics Analysis for Temperature Rise Prediction of power transformer. J. Electr Eng Technol, 8, 742-748.

Kellogg, R. A. (2000). The Delta- E effect in Terfenol-D and its application in a tunable mechanical resonator (master dissertation). Iowa State University: Ames, Iowa.

Moghaddam, N. S. et al. (2014). Enhancement of Bone Implants by Substituting Nitinol for Titanium (Ti-6Al-4V): A Modeling Comparison. in ASME 2014 Conference on Smart Materials, Adaptive Structures and Intelligent Systems. American Society of Mechanical Engineers.

Moghaddam, N. S. et al. (2016). Three Dimensional Printing of Stiffness-tuned, Nitinol Skeletal Fixation Hardware with an Example of Mandibular Segmental Defect Repair. Procedia CIRP, 49, 45-50.

Nurul, I., \& Samsuddin, S. A. (2013). Nurul Fadzlin Hasbullah, Siti Fauziah Toha, Parametric System Identification of Thermoelectric Cooler for Single Photon Avalanche Diode Application. Applied Sciences, Engineering and Technology, 6, 712-719.

Raad, B., Moghaddam, N. S., \& Elahinia, M. (2016). A numerical simulation of the effect of using porous superelastic Nitinol and stiff Titanium fixation hardware on the bone remodeling. in SPIE Smart Structures and Materials + Nondestructive Evaluation and Health Monitoring. International Society for Optics and Photonics.

Rahmanian, R. et al. (2014). Load bearing and stiffness tailored niti implants produced by additive manufacturing: A simulation study. in SPIE Smart Structures and Materials + Nondestructive Evaluation and Health Monitoring. International Society for Optics and Photonics.

Ravi, S., Annapragada, T. S., Paul, K., Marc, H., \& Suresh, V. G. (2012). Determination of electrical contact resistivity in Thermoelectric Modules (TEMs) from module-level measurements. IEEE Transactions on Components, Packaging and Manufacturing Technology, 2(4), 668-676.

Shayesteh, M. N. (2015). Toward Patient Specific Long Lasting Metallic Implants for Mandibular Segmental Defects. University of Toledo.

Wang, L. H. Y., Liu, Y. T., \& Yao, S. M. (2006). Analysis and Optimization for Uniformity of Magnetic Field. in Journal of Physics: Conference Series 48. Harbin 150001, China.

\section{Copyrights}

Copyright for this article is retained by the author(s), with first publication rights granted to the journal.

This is an open-access article distributed under the terms and conditions of the Creative Commons Attribution license (http://creativecommons.org/licenses/by/4.0/). 